\title{
DEVELOPMENT AND PRODUCTION OF HYBRID CIRCUITS FOR MICROWAVE RADIO LINKS
}

\author{
PER CHR. MALMIN \\ NERA AS, Division Bergen, PO Box 53, N-5032 MINDE, Norway
}

(Received May 30, 1977)

\begin{abstract}
During the last few years, the use of hybrid integrated circuits in microwave radio links has increased significantly. This paper reports on a range of microwave hybrid circuits now in production at our plant. These include transistor high power amplifiers, low noise mixers and various other circuits. The hybrid technology has proven to be cost competitive with the more conventional waveguide and coaxial technologies, and gives quite a few other advantages as well. Among these, the standardization of packages and modules is discussed in some detail. The trend is now to use the hybrid technology in VHF circuits as well. This opens up the possibility of integrating related functions in one package. The production cost in this case must be related to printed circuit boards, and it remains to be seen if there is any thing to gain in this respect. However the reduction of the size of modules and the time for circuit alignment will certainly give an improvement in the overall system cost. In order to reduce the cost of microwave hybrid circuits, thick film technology is currently being investigated for use up to $15 \mathrm{GHz}$. There is probably no specific upper frequency for thick film circuits, but the possibility of using conventional thick film technology depends on the actual circuits under consideration. Preliminary results of this work are reported, together with a cost comparison between thick film and thin film microwave hybrid circuits.
\end{abstract}

\section{INTRODUCTION}

This report will discuss the use of hybrid circuits in microwave radio links. It is mainly concerned with microwave hybrid circuits, using microstrip lines, and at first a survey of the principles of these circuits is given. Several examples of hybrid circuits currently in production at our company are shown. The use of VHF hybrid circuits, and the possibilities of integrating various SHF and VHF circuits are discussed. We will also report on investigations on thick film technology as a potentially cheaper way of making SHF hybrid circuits.

\section{MICROWAVE HYBRID CIRCUITS}

At microwave frequencies the wavelength is of the same order of magnitude as the circuit elements. As a consequence, one cannot simply connect diodes and transistors by conductors of arbitrary shape, they have to be interconnected through transmission lines of a specific characteristic impedance. Microstrip lines are the more often used transmission lines in hybrid circuits. When designing circuits with microstrip lines, the linewidth, or characteristic impedance, must be kept within close tolerances. The impedance of any semiconductor, packaged or in chip or beam lead form, must be matched to the $50 \mathrm{ohms}$ characteristic impedance of the microstrip line. This is done by using distributed circuit elements, i.e. sections of different transmission lines.

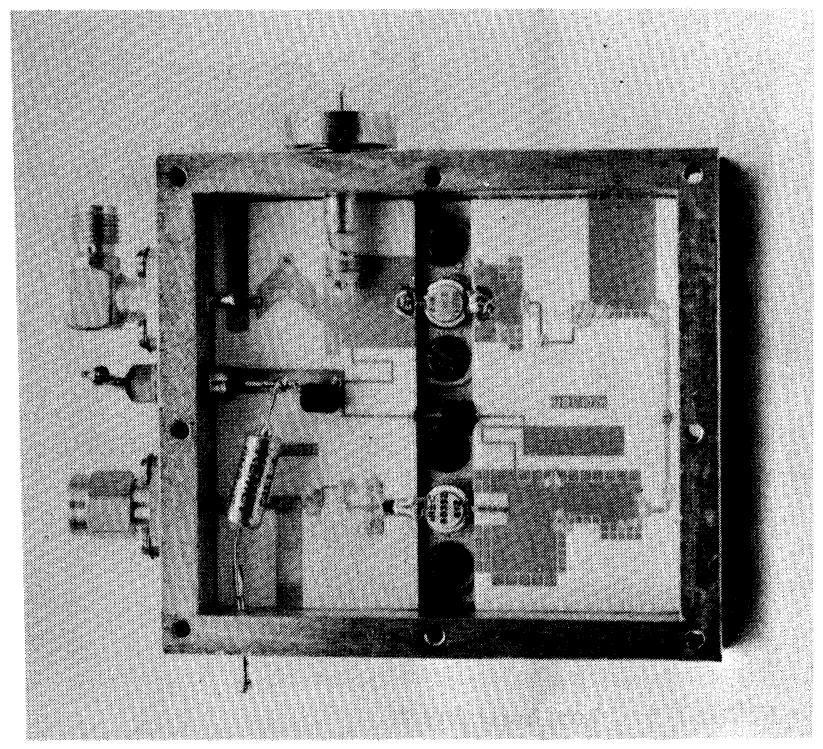

FIGURE 1 High power amplifier. Output power $5 \mathrm{~W}$, Bandwidth $10 \%$. Centre frequency $2 \mathrm{GHz}$. 


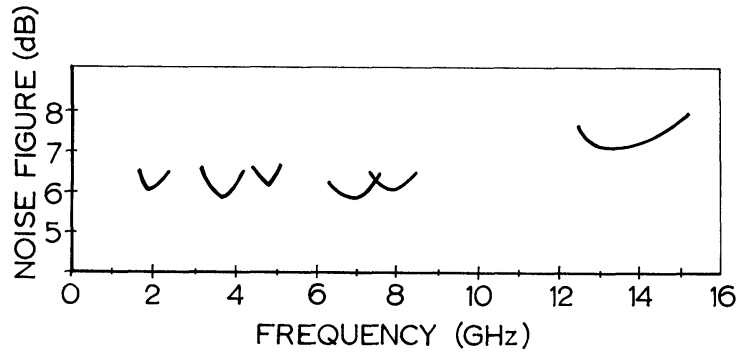

FIGURE 2 Noise figure of hybrid circuit mixers. $\mathrm{F}_{\mathrm{IF}}=70 \mathrm{MHz}, \mathrm{NF}_{\mathrm{IF}}=1.5 \mathrm{~dB}$

Use of thin film hybrid technology has many advantages when designing microwave circuits. For example:

- close dimensional control

- ease of component attachment, use of chips avoids problems due to package parasitics

- standardization of module size independent of frequency

- high reliability and reasonable cost

- size and weight reduction, important in mobile systems.

However, it must be kept in mind that not all circuit functions are suited for hybrid circuit technology. The most typical example is perhaps filters with high Q-value.

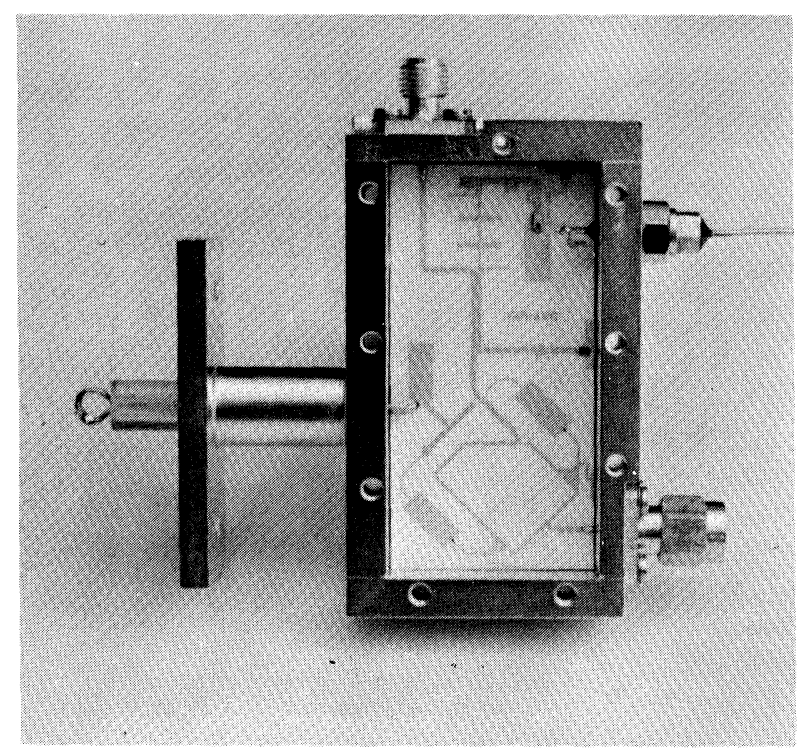

FIGURE 3 Low noise mixer.

$\mathrm{NF}=6 \mathrm{~dB}$ (incl. $1.5 \mathrm{~dB}$ IF noise). Frequency range RF \& LO: 3.4 to $4.2 \mathrm{GHz}$ IF: $70 \mathrm{MHz}$

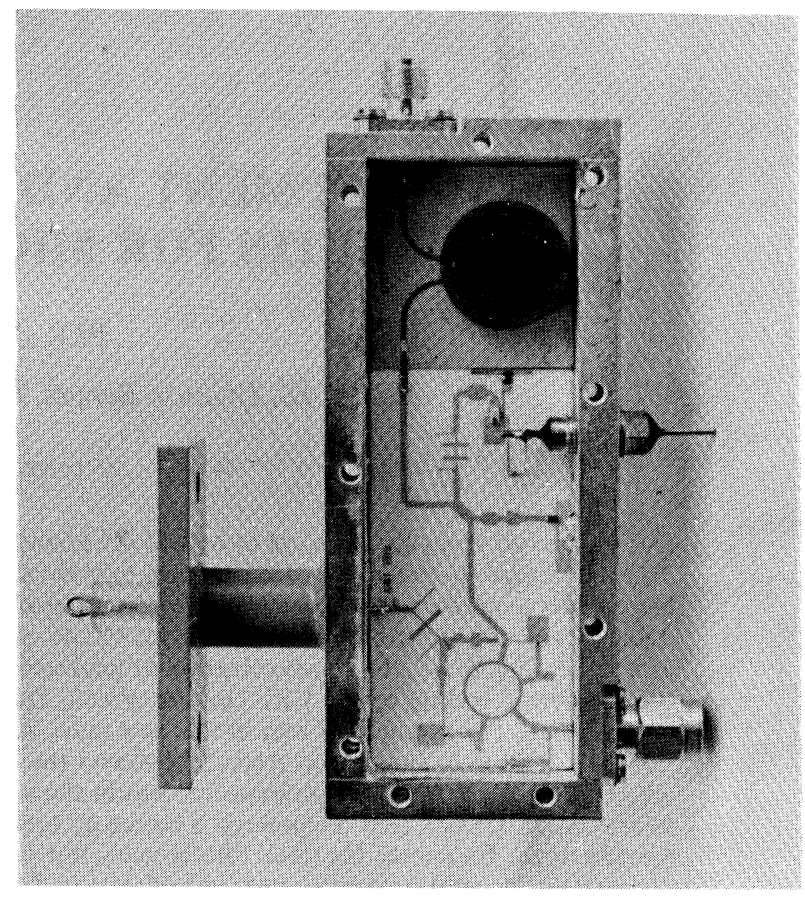

FIGURE 4 Low noise mixer with adjustable attenuator for LO power adjustment. Frequency range: RF \& LO: 7.4 to $8.4 \mathrm{GHz}$ IF: $70 \mathrm{MHz}$. NF $=6 \mathrm{~dB}$

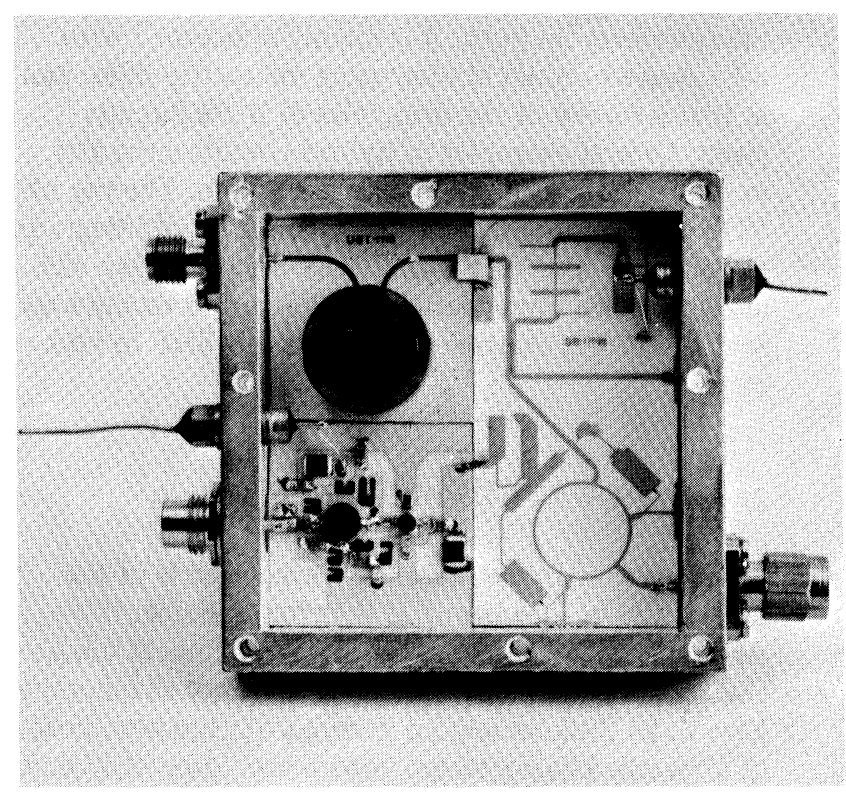

FIGURE 5 Integrated mixer and IF preamplifier, complete with adjustable attenuator and detector for $\mathrm{LO}$ power level. Frequency range: 4.4 to $5.0 \mathrm{GHz}$ 


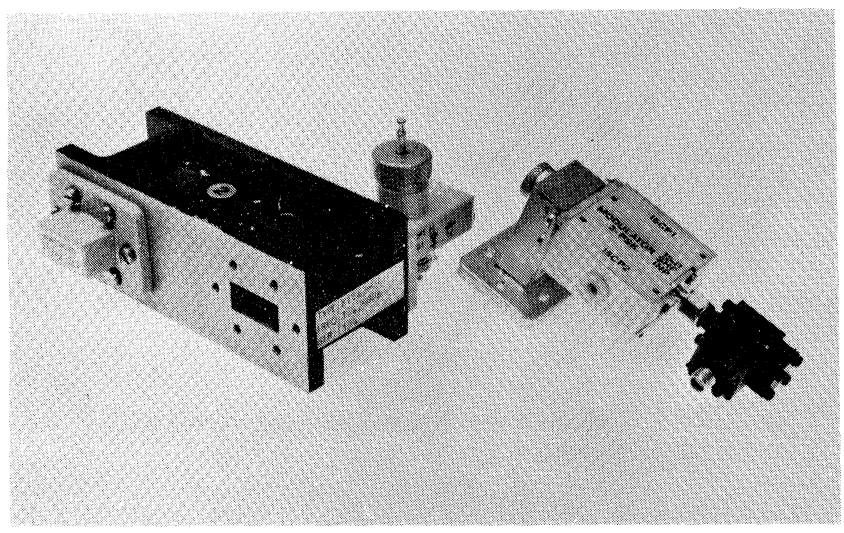

FIGURE 6 Two versions of a 2-PSK modulator. On the left hand side is the waveguide version, with a 4-port circulator. On the right hand side is the microstrip version, with a separate coaxial isolator.

The use of a computer in the circuit design may be extremely helpful. However, a serious limitation is the lack of available data for the semiconductors involved. High power devices are usually poorly characterized. This is at least partly due to the fact that impedance measurements on these devices may be more or less meaningless if performed at a power level and in a circuit very different from the actual application. Therefore, a somewhat pragmatic approach has to be taken in the design phase. One calculates an initial circuit layout using simplified models for the semiconductors involved. This circuit is then measured, and if necessary, changed by removing or adding metallization. These changes are often done by applying small amounts of silver conductive coating. This usually has the desired effect, because at microwave frequencies, every line or part of the conductor area, may be considered a circuit element.

Finally, the modifications are included in the gold metallization pattern by making a new mask.

\section{EXAMPLES OF MICROWAVE HYBRID CIRCUITS}

For our radio link transmitters we have developed high power, broadband transistor amplifiers, as shown in Figure 1.

Hybrid circuit technology has proved essential for the development of these amplifiers. Thin film gold, on alumina substrates, provides both over-theedge metallization for ground plane connections, and

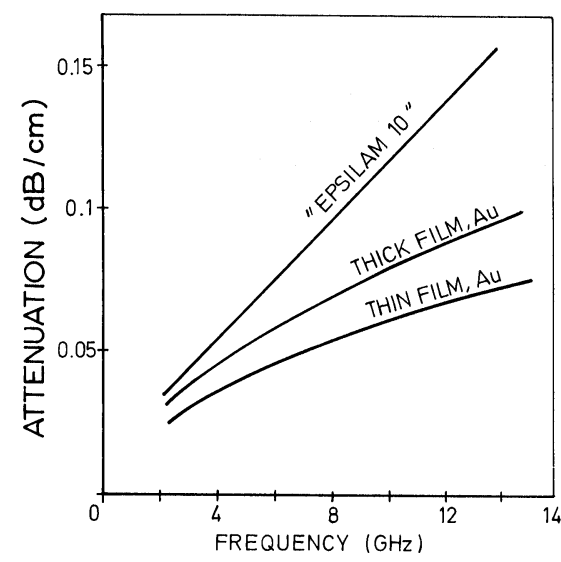

FIGURE 7 Attenuation in Microstrip lines with $50 \mathrm{ohms}$ characteristic impedance. Different technologies.

a favourable length to width ratio for low impedance lines. We are now producing a range of amplifiers with more than $5 \mathrm{~W}$ output power over a bandwidth of $10 \%$ at $2 \mathrm{GHz}$. The power transistors are not mounted on the substrates, but directly to the bottom of the box, in order to obtain proper heat sinking. It has been necessary to tune these amplifiers individually. This is done by locally applying a silver conductive coating while inspecting the frequency response on an oscilloscope. The tuned amplifier is next given a stabilization bake before final testing is made.

For radio link receivers, mixers with low noise figure must be used. We have developed a range of balanced microstrip mixers with noise figures as shown in Figure 2. Each circuit covers at least one radio frequency band. This broadband operation is obtained in spite of the fact that a $180^{\circ}$ "rat-race" coupler is used to separate the LO and RF signals.

A typical mixer circuit, operating over the frequency range from 3.4 to $4.2 \mathrm{GHz}$ is shown in Figure 3. The shape of this box was determined by the requirement that this mixer should replace an older waveguide mixer in an existing system. On the other hand, this made it possible to produce hybrid circuit low noise mixers, covering all the radio link bands from $3.4 \mathrm{GHz}$ and upwards, and all fitting this same box. Type HP 5082-2768 Beam-Lead Schottky diodes are used for all these circuits. In addition to the mixer, there is a detector circuit on the same substrate. This circuit is used to monitor the LO power level.

It is often convenient to have a variable attenuator for the LO power within the same box. 


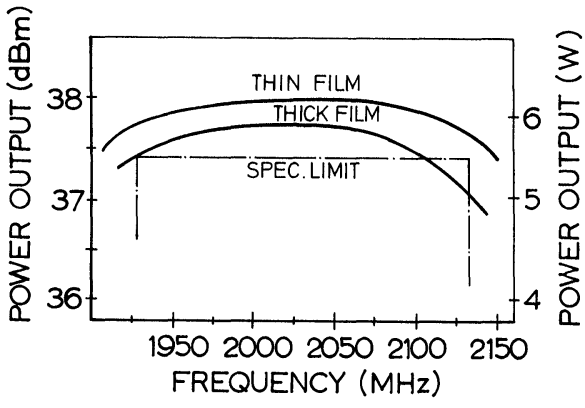

FIGURE 8 Frequency response of microstrip power amplifiers.

This attenuator is used to adjust the LO drive level to obtain minimum noise figure. With this attenuator, the complete circuit may look as shown in Figure 4, which is a mixer covering the 7.4 to $8.4 \mathrm{GHz}$ frequency range. At microwave frequencies one cannot use potentiometers, so the attenuator element is a ferrite disc which can be moved up and down above the microstrip line. The closer the ferrite is to the line, the greater the attenuation. This part of the circuit is produced on a PTFE based substrate with trade name Epsilam 10, which is cheaper than alumina and is easily machined with ordinary workshop equipment.

Figure 4 shows the general design of the boxes. We are using a U-shaped profile with 1 in. internal width. This profile may be cut to any desired length to accommodate 1,2 or 3 substrates. The box is completed by soldering two end walls to the profile, using a high temperature solder. The connectors may be located according to the application. Thus a high degree of standardization of the production process is obtained, while flexibility in circuit layout is maintained.

\section{INTEGRATION OF SHF AND VHF HYBRID CIRCUITS}

We have started to work with hybrid circuit technology in a special way, namely with circuits for microwave frequencies. We are now moving downwards in frequency, and hope to see advantages from integrating, for example a thin film mixer and a thick film IF-preamplifier in the same box. An example of this is shown in Figure 5. The requirements on the preamplifier are first of all a low noise figure of less than $1.5 \mathrm{~dB}$, a flat frequency response up to around

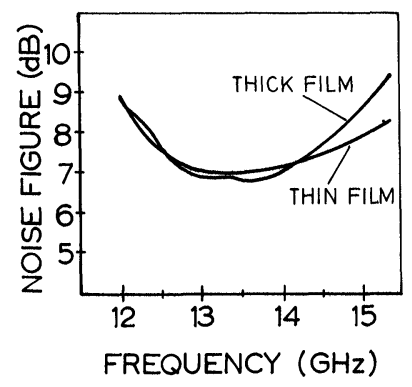

FIGURE 9 Mixer noise figure. The same circuit in thin and thick film technology.

$100 \mathrm{MHz}$, a gain of approximately $25 \mathrm{~dB}$ and output VSWR of less than 1.1. This goal has been achieved by using a 2 stage transistor amplifier with current feedback. Encapsulated transistors are used in order to simplify packaging and testing problems. Transistors, and chip capacitors and inductors, are soldered into the circuit.

This unit has several advantages. It requires a minimum of alignment, confines the lowest signal levels within one box, has a broadband frequency response, both SHF and VHF, and saves a lot of space in the equipment. One does not here see any cost saving in the production of the thick film amplifier compared to the former printed circuit board version. However, the reduction of system setup time is very important.

\section{POSSIBILITIES FOR COST REDUCTION}

A few possibilities for cost reduction with microwave hybrid circuits will be discussed.

One example of what has been obtained with thin film circuits, is a two-phase shift modulator for a $13 \mathrm{GHz}$ digital radio link, Figure 6. The original waveguide version, a reflection type circuit, had to use an invar cavity to get sufficient temperature stability. The new microstrip circuit is of the "Switched-line" type on alumina substrate. In production the microstrip circuit is approximately $25 \%$ cheaper than the waveguide version. This is primarily due to lower material costs. This cost advantage is obtained even if the hybrid circuit version of the modulator has four chip diodes while the waveguide version has only one diode.

Another possibility is using thick film technology for microwave hybrid circuits. Here two problems 
must be taken into consideration. The technical question if the line definition and other properties of thick films are good enough for microwave applications, and then the economical question if any cost reduction is really obtained.

Figure 7 shows loss per unit length of $50 \mathrm{ohm}$ microstrip lines made with different technologies. These data are calculated from measurements of Q-values of ring resonators. The difference between thin film and thick film gold conductors is about $20 \%$. The significance of this difference will depend on the application. It is also seen that the material "Epsilam 10" has higher losses than both thin and thick films.

Figure 8 shows the frequency response of thin film and thick film versions of the $2 \mathrm{GHz}$ power amplifiers. While the thin film units are well above the specification limits, the thick film units give a low power output at the higher frequencies.

Figure 9 shows the noise figure of receiver mixers in the frequency range 13 to $15 \mathrm{GHz}$. The noise figure is approximately the same for both the thin and thick film circuits, except at the higher frequencies. This has been shown to be due to mismatch, rather than ohmic losses in the thick film.

These examples indicate that it is not possible to state in general terms for instance a maximum frequency limit for using thick film technology.

Returning to the economical aspects of this problem, the result is totally dependent on the production volume. For a specific case, namely the cost of establishing a conductor pattern on the 1 in. $\times 2$ in. ceramic substrates for the $2 \mathrm{GHz}$ amplifiers previously mentioned, the comparison comes out as follows, for a production volume of a few hundred substrates:

Using thin films, this cost is approximately $\$ 25$ per substrate, of which the metallized substrate itself amounts to $\$ 18$.

NERA AS has still no printing facilities for thick film circuits, but has them made at the Central Institute for Industrial Research in Oslo. The total cost for a printed substrate is approximately $\$ 11$, thus a cost reduction of more than $50 \%$ is obtained compared to thin films.

However, in order to maintain this cost reduction through the rest of the production process, one must not run into thick film related problems later on with bonding, encapsulation or degraded electrical performance. This is why the microwave properties of various thick film pastes are now under investigation.

\section{CONCLUSION}

This report has tried to show that the use of hybrid circuit technology can be very advantageous in microwave radio links, even if the volue of production is quite small. There are at least two reasons for this conclusion. Firstly, regarding production costs, one does not compete with printed circuit boards, but with relatively complex waveguide or coaxial circuits. Secondly, the development or prototype lab needed, will have a considerable capacity, which proved sufficient for our production volume. 

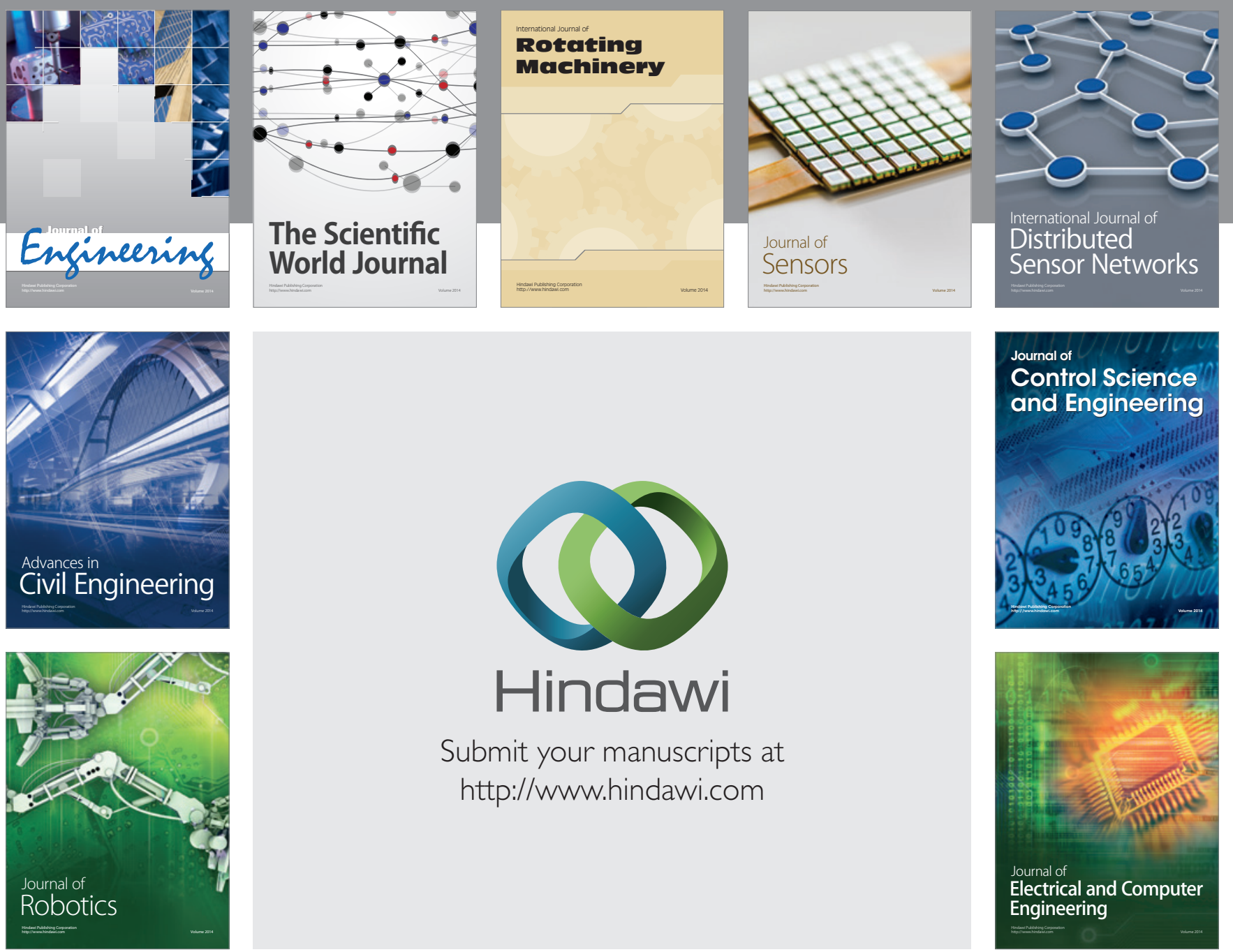

Submit your manuscripts at

http://www.hindawi.com
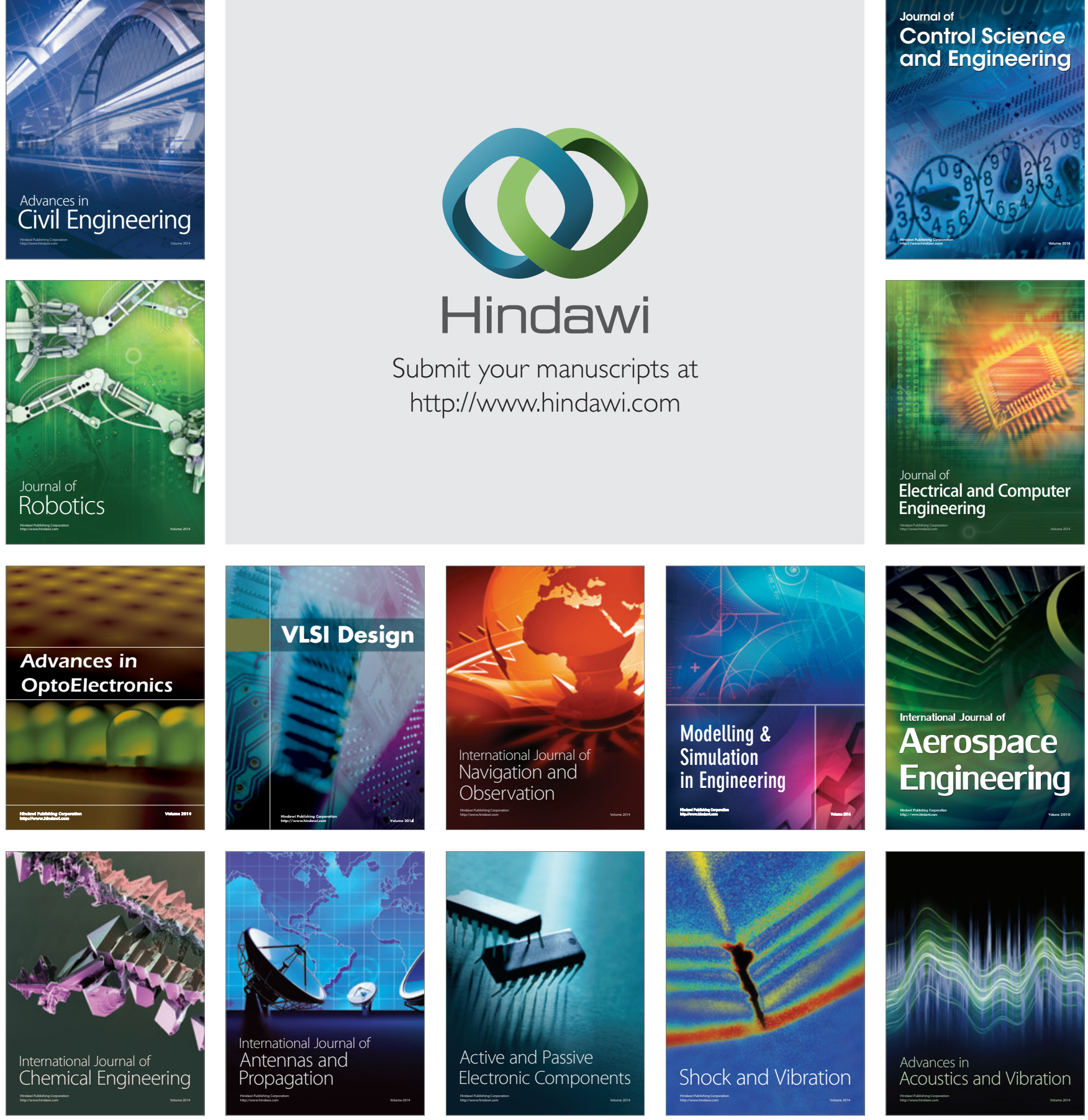